\title{
Cavity-enhanced photoacoustic sensor based on a whispering-gallery-mode diode laser
}

\author{
Yufeng Pan ${ }^{1,2}$, Lei Dong ${ }^{1,2}$, Hongpeng $\mathrm{Wu}^{1,2}$, Weiguang $\mathrm{Ma}^{1,2}$, Lei Zhang ${ }^{1,2}$, Wangbao Yin ${ }^{1,2}$, Liantuan Xiao ${ }^{1,2}$, \\ Suotang Jia ${ }^{1,2}$, and Frank K. Tittel ${ }^{3}$ \\ ${ }^{1}$ State Key Laboratory of Quantum Optics and Quantum Optics Devices, Institute of Laser Spectroscopy, \\ Shanxi University, Taiyuan 030006, China \\ ${ }^{2}$ Collaborative Innovation Center of Extreme Optics, Shanxi University, Taiyuan 030006, China \\ ${ }^{3}$ Department of Electrical and Computer Engineering, Rice University, Houston, Texas 77005, USA
}

Correspondence: Lei Dong (donglei@sxu.edu.cn)

Received: 30 December 2018 - Discussion started: 4 January 2019

Revised: 5 March 2019 - Accepted: 10 March 2019 - Published: 25 March 2019

\begin{abstract}
A cavity-enhanced photoacoustic (CEPA) sensor was developed based on an ultra-narrow linewidth whispering-gallery-mode (WGM) diode laser. A cavityenhanced photoacoustic module (CEPAM) was designed to match the output beam from the WGM-diode laser, resulting in an increase in the excitation light power, which, in turn, significantly enhanced the photoacoustic signal amplitude. The results show that a signal gain factor of 166 was achieved, which is in excellent agreement with the power enhancement factor of 175 after considering the power transmissivity. The performance of the sensor was evaluated in terms of the detection sensitivity and linearity. A $1 \sigma$ detection limit of $0.45 \mathrm{ppmV}$ for $\mathrm{C}_{2} \mathrm{H}_{2}$ detection was obtained at atmospheric pressure with a $1 \mathrm{~s}$ averaging time.
\end{abstract}

\section{Introduction}

Photoacoustic spectroscopy (PAS) is an important trace gas detection technique that is widely applied to atmospheric science, breath analysis and industrial process control (Siciliani et al., 2014; Wojtas et al., 2014; Yin et al., 2017a). In the PAS technique, modulated excitation light is selectively absorbed by a target gas and results in the generation of an acoustic wave by non-radiative energy relaxation processes. One of the unique PAS advantages is that its sensitivity is proportional to excitation laser power, and thus the performance of PAS sensors can be improved by increasing the excitation laser power. When a commercially avail- able telecommunication diode laser is employed as an excitation light source, a commonly used method to increase the laser power is to use an erbium-doped fiber amplifier (EDFA) to boost the output optical power (Chen et al., 2018; He et al., 2018; Peng et al., 2009). For example, in 2015, Wu et al. (2015) demonstrated a quartz-enhanced photoacoustic spectroscopy (QEPAS) based $\mathrm{H}_{2} \mathrm{~S}$ sensor operating at $1582 \mathrm{~nm}$ combined with an EDFA (Wu et al., 2015). With $\mathrm{a} \sim 1.4 \mathrm{~W}$ optical excitation power and $67 \mathrm{~s}$ averaging time, the $\mathrm{H}_{2} \mathrm{~S}$ detection sensitivity was reduced from several $\mathrm{ppmV}$ level down to $142 \mathrm{ppbV}$ in $\mathrm{N}_{2}$, which is the best value for the $\mathrm{H}_{2} \mathrm{~S}$ QEPAS sensors reported so far. Yin et al. (2017b) developed a conventional photoacoustic (CPA) sensor operating at the same wavelength for $\mathrm{H}_{2} \mathrm{~S}$ detection in $\mathrm{SF}_{6}$ by means of an EDFA and a background-gas-induced high- $Q$ photoacoustic cell (Yin et al., 2017b). A $1 \sigma$ detection limit of $109 \mathrm{ppbV}$ was achieved with a $1 \mathrm{~s}$ averaging time. An alternative method of achieving high power is to combine PAS with cavity-enhanced absorption spectroscopy (CEAS) (Hippler et al., 2010; Kachanov et al., 2013; Wojtas et al., 2017). In 2015, Patimisco et al. (2015) proposed an intra-cavity QEPAS (I-QEPAS) sensor for $\mathrm{CO}_{2}$ detection at $4.33 \mu \mathrm{m}(\mathrm{Pa}-$ timisco et al., 2015). A power enhancement factor of $\sim 240$ was achieved with an intracavity power of $\sim 0.72 \mathrm{~W}$, resulting in a minimum detection limit of $300 \mathrm{pptV}$ at a total gas pressure of 50 mbar with a $20 \mathrm{~s}$ integration time.

Cavity-enhanced absorption spectroscopy (CEAS) is based on the use of optical resonant cavities in order to enhance light interaction with a gas species inside the cavity 
(Gherman and Romanini, 2002; He et al., 2018; Yi et al., 2016). In CEAS setups, a proper locking between the laser wavelength and the cavity resonance mode must be carried out via two approaches: (i) the cavity length is controlled by a piezo transducer (PZT) for the resonance mode to follow laser wavelength; and (ii) the length of the cavity is fixed, and the laser wavelength is locked to the cavity resonance mode. When the locking between the laser wavelength and the fundamental optical mode of the cavity is realized, the power inside the cavity will be enhanced significantly by a power enhancement factor $G$, and the value of $G$ can be calculated according to Eq. (1):

$G=\frac{F}{\pi}$

where $F$ represents the finesse of the optical cavity.

In this manuscript, we developed a cavity-enhanced photoacoustic (CEPA) sensor system for acetylene $\left(\mathrm{C}_{2} \mathrm{H}_{2}\right)$ detection at $1530.98 \mathrm{~nm}$ based on an ultra-narrow linewidth WGM-diode laser. A Fabry-Perot $(F-P)$ cavity with a finesse of 550 was designed. The laser wavelength was locked to the $F-P$ cavity mode by a Pound-Drever-Hall (PDH) locking technique (Black, 2000; Drewer et al., 1983) since the WGM-diode laser linewidth is far narrower than that of the cavity mode. A differential photoacoustic cell with two electret condenser cylindrical microphones was designed to detect the photoacoustic signal. To enhance the photoacoustic signal, the photoacoustic cell was inserted between the two cavity mirrors. The performance of the CEPA sensor was compared with a CPA sensor without cavity and evaluated at different $\mathrm{C}_{2} \mathrm{H}_{2}$ concentration levels.

\section{Sensor design}

\subsection{Characterization of ultra-narrow linewidth WGM-diode laser}

The portable WGM-diode laser (OEwaves, USA, OE4023153160-PA02-FM100) has an ultra-narrow linewidth of $<200 \mathrm{~Hz}$ for $10 \mu \mathrm{s}$ and good tunability in the wavelength range of 1529 to $1532 \mathrm{~nm}$ with an output power of $4 \mathrm{~mW}$ after stabilizing. The laser frequency can be tuned mode hopfree over $50 \mathrm{GHz}$ at a tuning rate of $1 \mathrm{GHz} \mathrm{s}^{-1}$ by changing the temperature of the laser internal resonator and over $1 \mathrm{GHz}$ at a tuning rate of $40 \mathrm{MHz} \mu \mathrm{s}^{-1}$ by an external voltage to change the length of an internal piezoelectric crystal. Figure 1 shows the tunable wavelength range of the WGMdiode laser for seven different wavelength bands when the laser temperature was fixed and the internal piezoelectric crystal was changed by an external voltage. Due to the $\sim$ $1 \mathrm{GHz}$ limit for each wavelength band, the tunable range cannot link up end-to-end. The laser beam was collimated by a fiber-coupled collimator (OZ Optics, Canada, HPUCOT.3A-1550-P-2AS). The output beam quality from the collimator was evaluated by a scanning-slit optical beam profiler

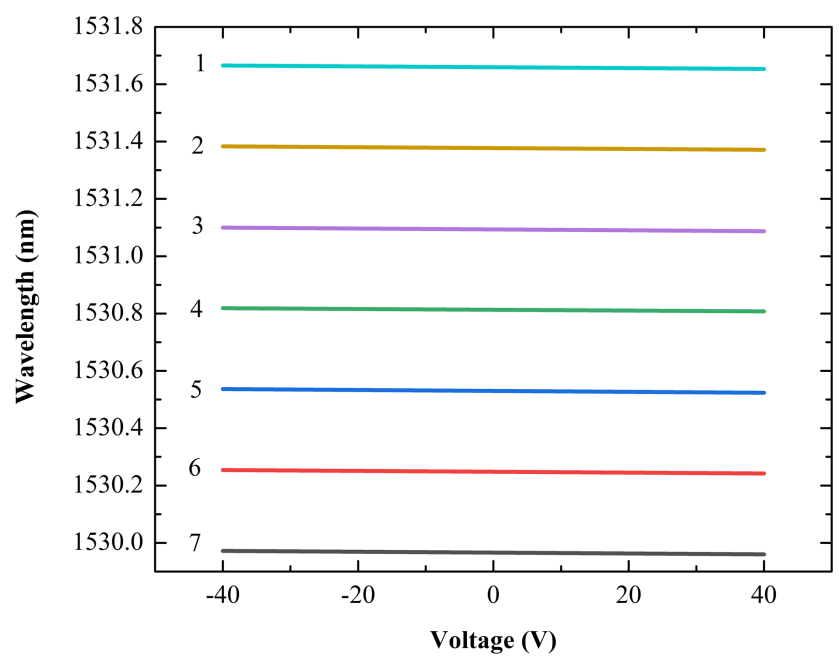

Figure 1. Tunable wavelength range of the WGM-diode laser for seven different wavelength bands when the laser temperature was fixed and the internal piezoelectric crystal was changed by an external voltage.

(THORLABS, USA, BP209-IR2/M). Figure 2a and b show the two-dimensional intensity distribution of the laser spot and the three-dimensional laser beam profile, respectively, at $15.2 \mathrm{~cm}$ from the collimator. The laser beam exhibits an excellent Gaussian fundamental mode with a spot size of $860 \mu \mathrm{m}$.

\subsection{Design of the cavity-enhanced photoacoustic module}

A cavity-enhanced photoacoustic module (CEPAM) consists of a differential photoacoustic cell, a $F-P$ cavity, and a gas chamber with a gas inlet and outlet. The differential photoacoustic cell was designed as shown in Fig. 3a, which resembles the well-known differential Helmholtz resonator (Starecki and Geras, 2014; Zeninari et al., 1999; Zheng et al., 2017). It has two identical $90 \mathrm{~mm}$ parallel tube-shaped channels with diameters of $8 \mathrm{~mm}$ as two acoustic resonators. Two buffer volumes with lengths of $10 \mathrm{~mm}$ and diameters of $20 \mathrm{~mm}$ connect to the two channels at both ends, thus making the two channels act as acoustic open-open resonators and create a total optical absorption length of $110 \mathrm{~mm}$. This allows the beam from the WGM-diode laser to pass through the differential photoacoustic cell easily. When the laser intensity is modulated at the resonance frequency of the photoacoustic cell, a standing sound wave generated with the absorption of a target gas has its maximum acoustic pressure in the middle of the acoustic resonator. Hence, two selected electret condenser cylindrical microphones which have the same frequency response sensitivities are installed on the walls in the middle of each resonator to detect the acoustic pressure. The gas flow noise and external acoustic disturbances can be effectively suppressed by using a custom 
(a)

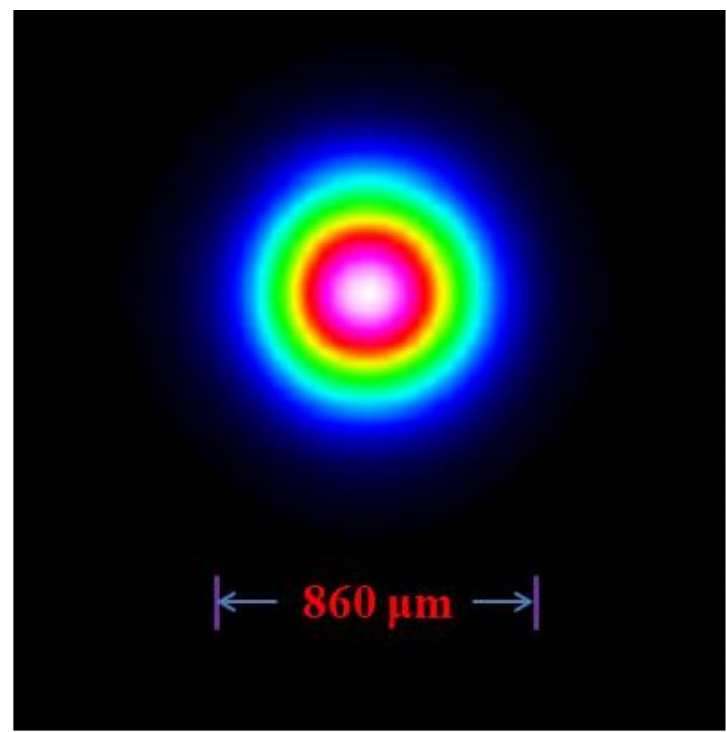

(b)

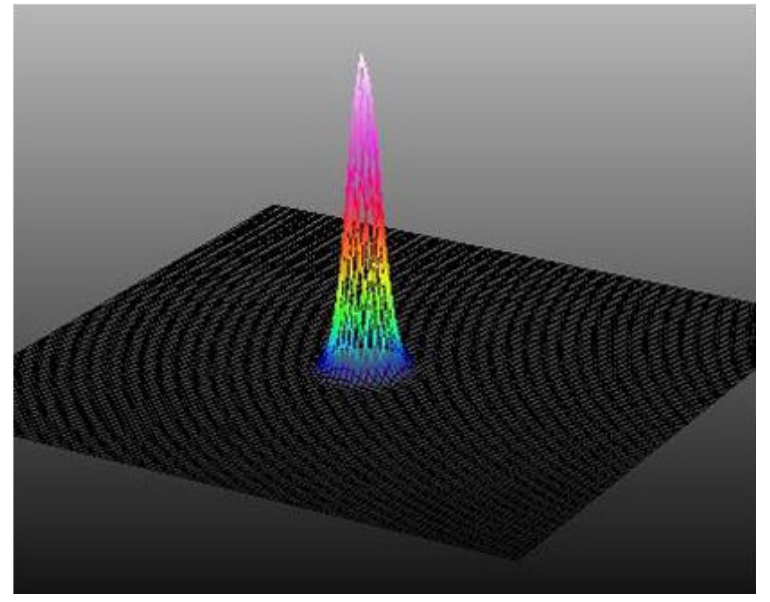

Figure 2. (a) The two-dimensional intensity distribution of the laser spot. (b) Three-dimensional laser beam profile.

transimpedance differential preamplifier. The signal coming from the microphone located in the acoustic resonator not illuminated by the laser beam is subtracted from the one related to the microphone located in the excited resonator and the resulting signal is subsequently amplified. Therefore, the performance of the PAS cell is improved. The resonance frequency of the differential photoacoustic cell in air was experimentally determined to be $f_{0}=1781.0 \mathrm{~Hz}$ and the full width at half maximum (FWHM) of the frequency response curves (resonance width) was $\Delta f=40 \mathrm{~Hz}$, corresponding to a quality factor $Q=f_{0} / \Delta f=45$.

Figure $3 \mathrm{~b}$ illustrates the schematic of the CEPAM. The $F-P$ cavity consists of a $25.4 \mathrm{~mm}$ plane mirror as the incident mirror and a $25.4 \mathrm{~mm}$ plane-concave mirror with a $1 \mathrm{~m}$ radius of curvature as the exit mirror. The two mirrors were coated with a high reflective coating of 0.995 . The cavity length was $160 \mathrm{~mm}$, which was longer than the $110 \mathrm{~mm}$ length of the differential photoacoustic cell. The designed (a)

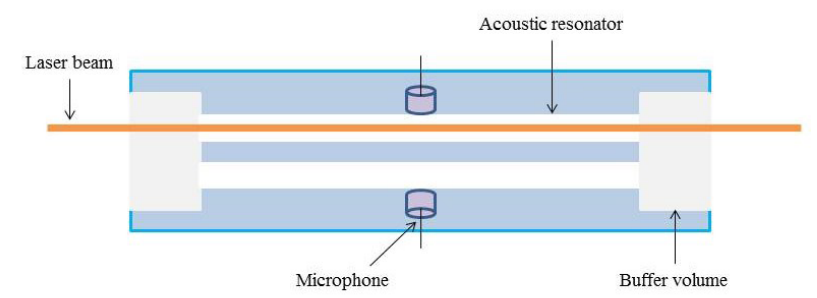

(b)

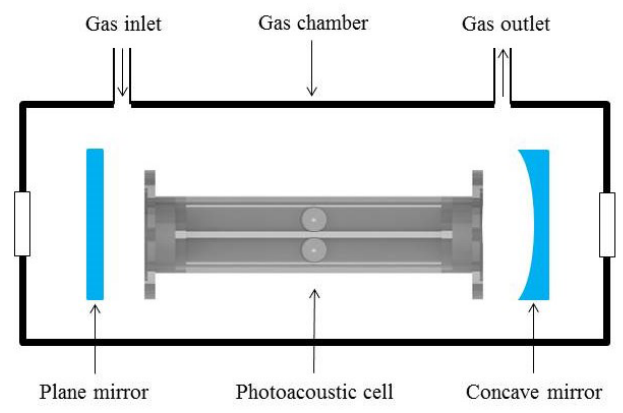

Figure 3. (a) Schematic diagram of the differential photoacoustic cell. (b) Schematic diagram of the cavity-enhanced photoacoustic module (CEPAM).

differential photoacoustic cell was inserted between the two cavity mirrors as shown in Fig. 3b. A gas chamber made of polymethyl methacrylate with a gas inlet and outlet was fabricated and used to provide environmental stabilization of the cavity.

\section{Experimental setup of the sensor system}

The experimental setup of the CEPA sensor system is depicted in Fig. 4. A mode-matching lens (L1), a half-wave plate $(\lambda / 2)$, a polarization beam splitter (PBS) and a quarterwave plate $(\lambda / 4)$ were placed in front of the cavity. Two focusing lenses (L2 and L3) were used to focus the transmitted and reflected lights from the cavity onto the photodiode detectors (PD1 and PD2) (THORLABS, USA, PDA10CF-EC), respectively. In order to achieve the mode matching, a ramp signal was generated by the first function signal generator (SG1) (Agilent, USA, Model 33500B) to scan the laser wavelength. The transmitted signal was detected by the PD1 and recorded by an oscilloscope (Tektronix, USA, DPO 2024). The second function signal generator (SG2) (Tektronix, USA, AFG 3102) generated two sine-wave signals with the same frequency $(15 \mathrm{MHz})$ and an adjustable phase difference. One of them was applied to a fiber-coupled electrooptic modulator ( $f$-EOM) (Keyang photonics, China, KYPM-1550-10-PP-FA) to modulate the laser wavelength. Another was directed to the mixer (Mini-Circuits, USA, ZLW-1) and mixed with the reflected signal that was reflected by the PBS to the PD2. A low-pass filter with an upper cutoff frequency of $1 \mathrm{MHz}$ (Stanford Research Systems, USA, Model 


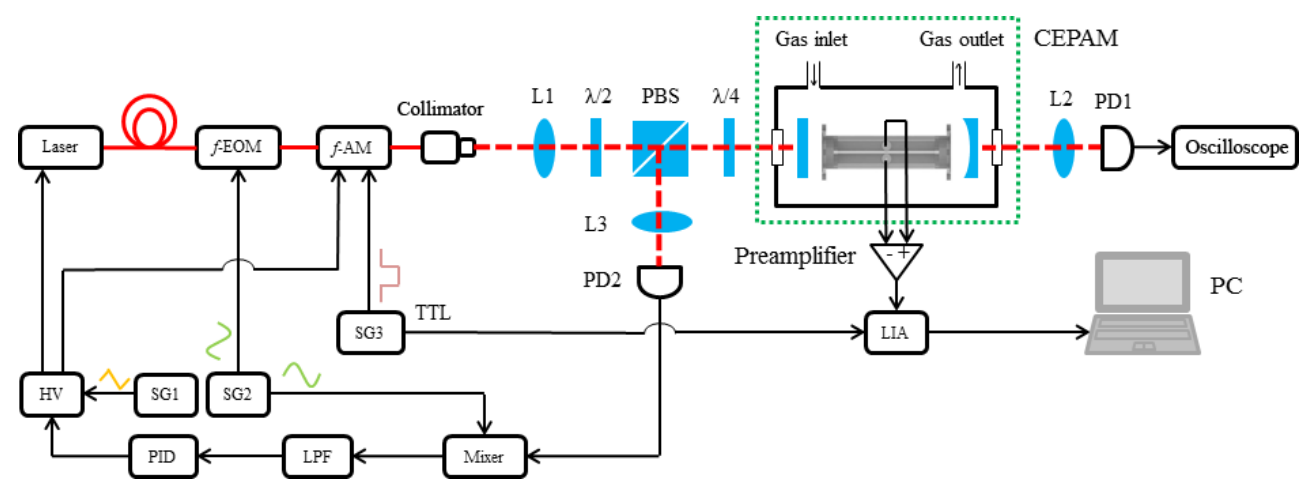

Figure 4. Experimental setup of the CEPA sensor system: $f$-EOM, fiber-coupled electro-optic modulator; $f$-AM, fiber-coupled amplitude modulator; L1, mode-matching lens; $\lambda / 2$, half-wave plate; PBS, polarization beam splitter; $\lambda / 4$, quarter-wave plate; L2 and L3, focusing lenses; PD, photodiode detector; SG, function signal generator; LPF, low-pass filter; PID, proportional-integral-derivative controller; HV, high-voltage amplifier; LIA, lock-in amplifier; PC, personal computer.
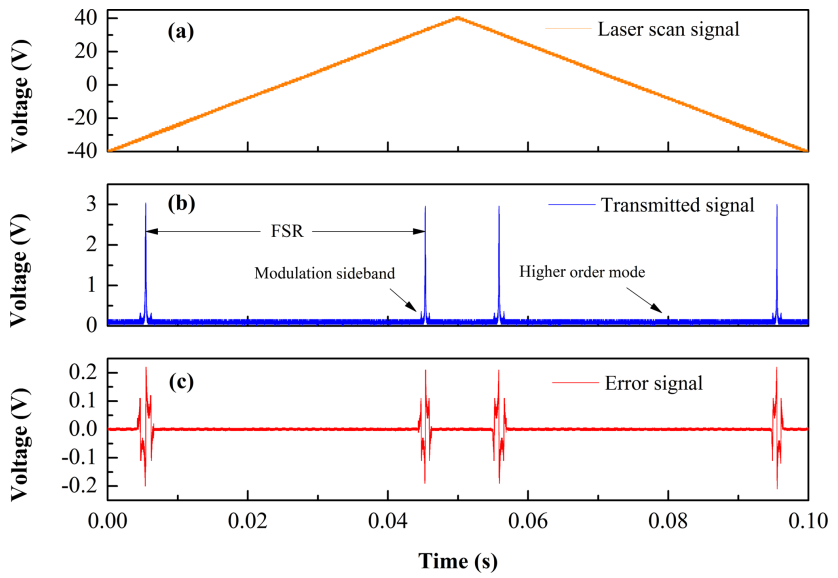

Figure 5. (a) Laser scan signal. (b) Transmitted signal from the cavity. (c) Error signal from the low-pass filter following the mixer.

SR560) was used after the output of the mixer to acquire a low-frequency signal as an error signal. The error signal was directed to a proportional-integral-derivative (PID) controller (Stanford Research Systems, USA, Model SIM960), which can provide a control signal to adjust the laser wavelength locking it to the cavity mode.

To sweep over a full cavity free-spectral range (FSR) of $\sim 0.9 \mathrm{GHz}$, a $10 \mathrm{~Hz}$ ramp signal was applied to the WGMdiode laser, as shown in Fig. 5a. The transmitted signal and the error signal were recorded using the oscilloscope as shown in Fig. 5b and c, respectively. Based on the FSR and the linewidth of the cavity $(\Delta v)$ from Fig. 5b, the finesse $F$ $(\mathrm{FSR} / \Delta v)$ is 550 . Therefore, a power enhancement factor $G$ of 175 is obtained according to Eq. (1), which means that the detection sensitivity of PAS can be improved by 175 times.

To verify the improved performance of the CEPA sensor system based on the WGM-diode laser, a $\mathrm{C}_{2} \mathrm{H}_{2}$ absorption line located at $1530.98 \mathrm{~nm}$ with an intensity of $4.00 \times$ $10^{-21} \mathrm{~cm}$ molecule ${ }^{-1}$ was selected as a target line. The wave-

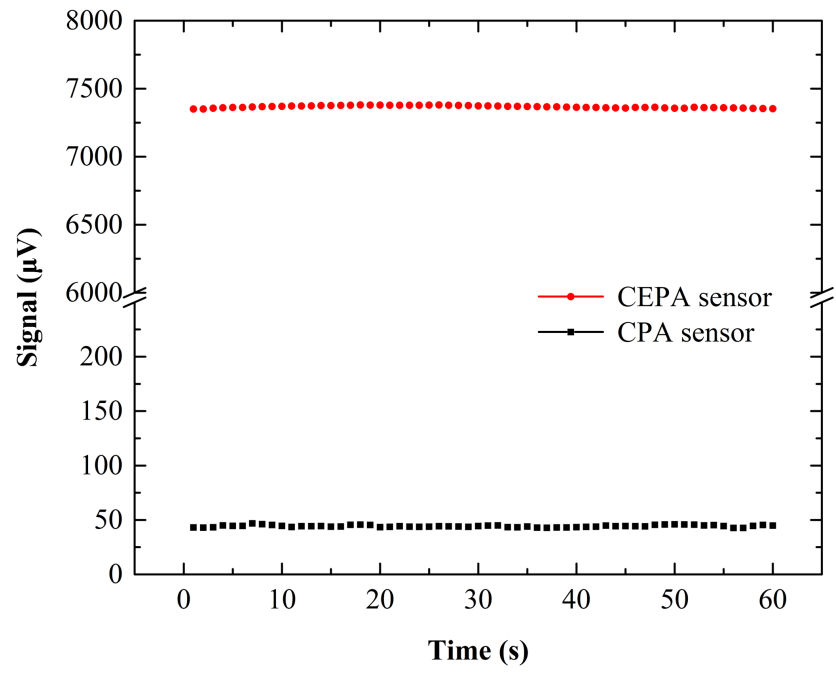

Figure 6. Comparison between the CEPA and CPA signal amplitudes from a $500 \mathrm{ppmV} \mathrm{C}_{2} \mathrm{H}_{2} / \mathrm{N}_{2}$ gas mixture.

length of the WGM-diode laser was tuned to the target line by means of a wavelength meter (HighFinesse, Germany, WS6). The laser wavelength was locked to the cavity mode. In order to generate a photoacoustic signal, a fiber-coupled amplitude modulator $(f$-AM) (Photline Technologies, France, MX-LN-10) with a DC bias generated by a high-voltage amplifier (Piezomechanik GmbH, Germany, SVR 200-3) and a square wave of $50 \%$ duty cycle generated by the SG3 (Tektronix, USA, AFG 3102), was employed to modulate the laser intensity before the laser beam entered the collimator. The $f$-AM can provide a DC extinction ratio of $20 \mathrm{~dB}$, which can meet the requirement of the intensity modulation. The frequency of the square wave was $1781.0 \mathrm{~Hz}$, corresponding to the resonance frequency of the differential photoacoustic cell. The loss of the $f$-EOM and the $f$-AM results in a final incident power of $0.7 \mathrm{~mW}$ in front of the $F-P$ cavity. The 

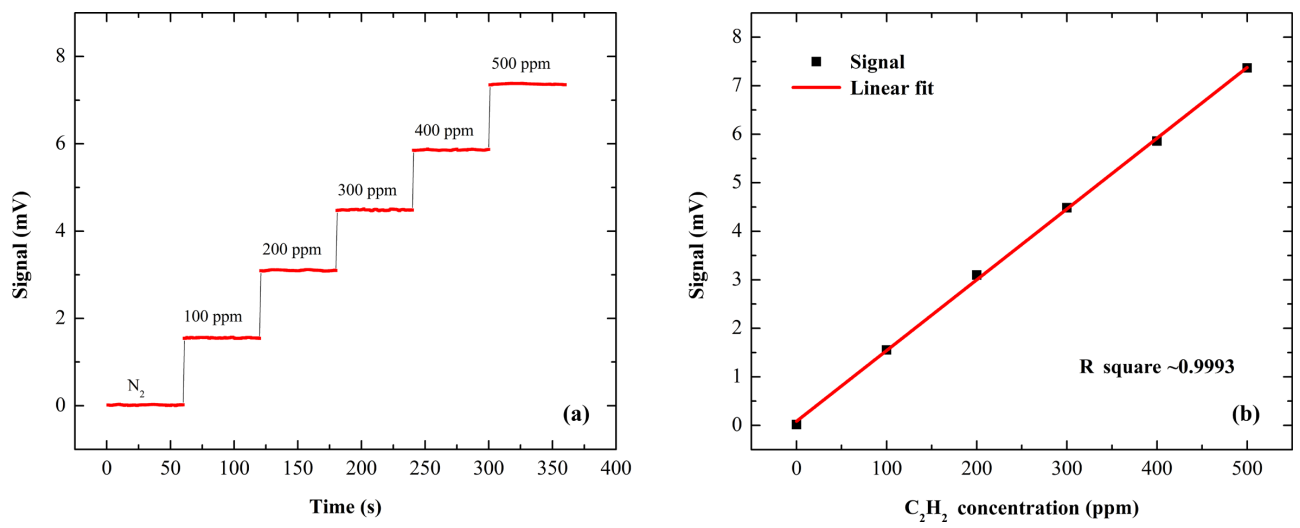

Figure 7. (a) CEPA signal at the different $\mathrm{C}_{2} \mathrm{H}_{2}$ concentration levels. (b) Linearity of the CEPA sensor system.

photoacoustic signal from the differential preamplifier was fed into a lock-in amplifier (LIA) (Stanford Research Systems, USA, Model SR830), which demodulated the signal in the $1-f$ mode. The reference signal for the LIA was from the TTL signal output of SG3. A $12 \mathrm{~dB} /$ oct filter slope and a $1 \mathrm{~s}$ time constant were set for the LIA, corresponding to a detection bandwidth of $\Delta f=0.25 \mathrm{~Hz}$. The demodulated signal from the LIA was recorded by a personal computer and the data were processed with a LabVIEW software program. A certified $500 \mathrm{ppmV}_{2} \mathrm{H}_{2}$ gas cylinder was used. The different concentrations of $\mathrm{C}_{2} \mathrm{H}_{2} / \mathrm{N}_{2}$ gas mixtures were produced by a gas dilution system (Environics Inc., USA, Model EN4040).

\section{Results and discussion}

The CEPAM was first filled with $500 \mathrm{ppmV}_{2} \mathrm{H}_{2}$. The measurements were carried out at atmospheric pressure and room temperature. The signal amplitudes from the CEPA sensor system are shown in Fig. 6. As a comparison, the signal amplitudes from a CPA sensor without the $F-P$ cavity are also shown in Fig. 6. The CEPA sensor effectively enhanced the signal amplitude from 44.3 to $7366.8 \mu \mathrm{V}$, corresponding to a signal gain factor of 166 . Considering the ratio of the powers of incident and transmitted lights is $\sim 95 \%$ for the $F-P$ cavity, the power enhancement factor verified by PAS is 175 , which is in excellent agreement with the anticipated value.

In order to evaluate the performance of the CEPA sensor system in terms of minimum detection limit and linearity, pure $\mathrm{N}_{2}$ and five different concentration levels of the $\mathrm{C}_{2} \mathrm{H}_{2} / \mathrm{N}_{2}$ gas mixtures varying from 100 to $500 \mathrm{ppmV}$ were fed into the CEPAM. The sensor system was operated at atmospheric pressure and at room temperature. Sixty data points of the CEPA signal were recorded continuously with a $1 \mathrm{~s}$ averaging time at each concentration level as shown in Fig. 7a. With pure $\mathrm{N}_{2}$, the $1 \sigma$ noise level was found to be $6.6 \mu \mathrm{V}$. The scatter of consecutive measurements at a certain concentration level did not depend on the concentra- tion and was in agreement with pure $\mathrm{N}_{2}$. For a $500 \mathrm{ppmV}$ $\mathrm{C}_{2} \mathrm{H}_{2} / \mathrm{N}_{2}$ gas mixture, a signal amplitude of $7366.8 \mu \mathrm{V}$ was observed, and hence a signal-to-noise ratio (SNR) of 1110 can be achieved which corresponds to a minimum detection limit $(1 \sigma)$ of $0.45 \mathrm{ppmV}$. The plot in Fig. 7b is a representation of the same measurements after 60 sensor readings of each concentration step are averaged. This plot confirms the linearity of the sensor response to a concentration with a $R^{2}$ value of $>0.9993$.

In 2017, Ma et al. (2017) used QEPAS technique to detect $\mathrm{C}_{2} \mathrm{H}_{2}$ and obtain a detection sensitivity of $33.2 \mathrm{ppb}$ (Ma et al., 2017), which is 13 times better than this CEPA sensor. The possible reasons are the following. (1) The CEPA sensor employed the acetylene absorption line located at $6531.76 \mathrm{~cm}^{-1}$, which was different from the Yufei Ma line at $6534.37 \mathrm{~cm}^{-1}$. The line strength of $6531.76 \mathrm{~cm}^{-1}$ is $\sim 3$ times lower than that of $6534.37 \mathrm{~cm}^{-1}$. (2) The cavity mode was not moved to the top of the absorption line. In this way, the $\mathrm{C}_{2} \mathrm{H}_{2}$ spectral wing was detected, which resulted in a sensitivity loss of $\sim 3$ times. (3) The effective optical power in the optical cavity was $116 \mathrm{~mW}$. But Ma et al. (2017) used an EDFA to boost the laser power to $1500 \mathrm{~mW}$. In fact, the signal enhancement achieved from the cavity is more important in this research. A comparative detection limit can be expected after selecting the same absorption line and setting the cavity mode to the top of the absorption line.

The CEPA technique is very different from the CPA technique. It is true that the use of an optical cavity makes the sensor system more complicated. However, the CEPA technique has the potential to further improve the detect limit if a higher finesse cavity is employed since the detection sensitivity is proportional to the excitation optical power.

\section{Conclusions}

A CEPA sensor system based on a WGM-diode laser was demonstrated. The WGM-diode laser has an ultra-narrow linewidth and was used as the excitation source. A cavity- 
enhanced photoacoustic module was designed to enhance the laser power density inside the optical cavity and the photoacoustic cell, resulting in a signal gain factor of 166, when the laser wavelength was locked on the fundamental optical mode of the $F-P$ cavity by a PDH-locking technique. The combination of the cavity-enhanced absorption spectroscopy and photoacoustic spectroscopy can improve the excitation light power effectively, leading to a significant gain of the photoacoustic signal amplitude. The use of the fiber-coupled elements and the WGM-diode laser have the potential to develop a compact CEPA sensor system with a higher detection sensitivity with respect to the CPA sensor system. A further improvement of the CEPA sensor system can be achieved by using a higher finesse cavity or a higher power laser source.

Supplement. The supplement related to this article is available online at: https://doi.org/10.5194/amt-12-1905-2019-supplement.

Data availability. Data used for this study can be found in the Supplement.

Author contributions. YP and LD contributed the central idea, analyzed the data, and wrote the initial draft of the paper. HW developed the idea of the study. The remaining authors contributed to refining the ideas, carrying out additional analyses and finalizing this paper.

Competing interests. The authors declare that they have no conflict of interest.

Special issue statement. This article is part of the special issue "Advances in cavity-based techniques for measurements of atmospheric aerosol and trace gases". It is not associated with a conference.

Acknowledgements. Lei Dong acknowledges support by the National Key R\&D Program of China (2017YFA0304203), the National Natural Science Foundation of China (NSFC) (61622503, 61575113, 61805132, 11434007), the Changjiang Scholars and Innovative Research Team in University of Ministry of Education of China (IRT_17R70), the 111 project (D18001), the Outstanding Innovative Teams of Higher Learning Institutions of Shanxi, the Foundation for Selected Young Scientists Studying Abroad, Sanjin Scholar (2017QNSJXZ-04) and Shanxi “1331KSC". Frank K. Tittel acknowledges support by the US National Science Foundation (NSF) ERC MIRTHE award and the Robert Welch Foundation (grant no. C0568).

Review statement. This paper was edited by Weidong Chen and reviewed by two anonymous referees.

\section{References}

Black, E. D.: An introduction to pound-drever-hall laser frequency stabilization, Am. J. Phys., 69, 79-87, 2000.

Chen, K., Gong, Z., and Yu, Q.: Fiber-amplifier-enhanced resonant photoacoustic sensor for sub-ppb level acetylene detection, Sensor. Actuat. A Phys., 274, 184-188, 2018.

Drewer, R. W. P., Hall, J. L., Kowalski, F. V., Hough, J., Ford, F. M., Munley, A. J., and Ward, H.: Laser phase and frequency stabilization using an optical resonator, Appl. Phys. B, 31, 97-105, 1983.

Gherman, T. and Romanini, D.: Mode-locked cavity- enhanced absorption spectroscopy, Opt. Express, 10, 1033-1042, 2002.

He, Q., Zheng, C., Lou, M., Ye, W., Wang, Y., and Tittel, F. K.: Dual-feedback mid-infrared cavity-enhanced absorption spectroscopy for $\mathrm{H}_{2} \mathrm{CO}$ detection using a radio-frequency electrically-modulated interband cascade laser, Opt. Express, 26, 15436-15444, 2018.

He, Y., Ma, Y., Tong, Y., Yu, X., and Tittel, F. K.: HCN ppt-level detection based on a QEPAS sensor with amplified laser and a miniaturized 3D-printed photoacoustic detection channel, Opt. Express, 26, 9666-9675, 2018.

Hippler, M., Mohr, C., Keen, K. A., and Mcnaghten, E. D.: Cavityenhanced resonant photoacoustic spectroscopy with optical feedback cw diode lasers: a novel technique for ultratrace gas analysis and high-resolution spectroscopy, J. Chem. Phys., 133, 044308, https://doi.org/10.1063/1.3461061, 2010.

Kachanov, A., Koulikov, S., and Tittel, F. K.: Cavity-enhanced optical feedback-assisted photo-acoustic spectroscopy with a 10.4 $\mu \mathrm{m}$ external cavity quantum cascade laser, Appl. Phys. B, 110, 47-56, 2013.

Ma, Y., He, Y., Zhang, L., Yu, X., Zhang, J., Sun, R., and Tittel, F. K.: Ultra-high sensitive acetylene detection using quartzenhanced photoacoustic spectroscopy with a fiber amplified diode laser and a $30.72 \mathrm{kHz}$ quartz tuning fork, Appl. Phys. Lett., 110, 031107, https://doi.org/10.1063/1.4974483, 2017.

Patimisco, P., Borri, S., Galli, I., Mazzotti, D., Giusfredi, G., Akikusa, N., Yamanishi, M., Scamarcio, G., Natale, P. D., and Spagnolo, V.: High finesse optical cavity coupled with a quartz-enhanced photoacoustic spectroscopic sensor, Analyst, 140, 736-743, 2015.

Peng, Y., Zhang, W., Li, L., and Yu, Q.: Tunable fiber laser and fiber amplifier based photoacoustic spectrometer for trace gas detection, Spectrochim Acta. A, 74, 924-927, 2009.

Siciliani, D. C. M., Viciani, S., Borri, S., Patimisco, P., Sampaolo, A., Scamarcio, G., Natale, P. D., D’Amato, F., and Spagnolo, V.: Widely-tunable mid-infrared fiber-coupled quartz-enhanced photoacoustic sensor for environmental monitoring, Opt. Express, 22, 28222-28231, 2014.

Starecki, T. and Geras, A.: Differential open photoacoustic helmholtz cell, Int. J. Thermophys., 35, 2259-2268, 2014.

Wojtas, J., Tittel, F. K., Stacewicz, T., Bielecki, Z., Lewicki, R., Mikolajczyk, J., Nowakowski, M., Szabra, D., Stefanski, P., and Tarka, J.: Cavity-Enhanced Absorption Spectroscopy and Photoacoustic Spectroscopy for Human Breath Analysis, Int. J. Thermophys., 35, 2215-2225, 2014.

Wojtas, J., Gluszek, A., Hudzikowski, A., and Tittel, F. K.: MidInfrared Trace Gas Sensor Technology Based on Intracavity Quartz-Enhanced Photoacoustic Spectroscopy, Sensors, 17, 513/1-513/9, 2017. 
Wu, H., Dong, L., Zheng, H., Liu, X., Yin, X., Ma, W., Zhang, L., Yin, W., Jia, S., and Tittel, F. K.: Enhanced near-infrared QEPAS sensor for sub-ppm level $\mathrm{H}_{2} \mathrm{~S}$ detection by means of a fiber amplified $1582 \mathrm{~nm}$ DFB laser, Sensor. Actuat. B, 221, 666-672, 2015.

Yi, H., Wu, T., Wang, G., Zhao, W., Fertein, E., Coeur, C., Gao, X., Zhang, W., and Chen, W.: Sensing atmospheric reactive species using light emitting diode by incoherent broadband cavity enhanced absorption spectroscopy, Opt. Express, 24, A781-A790, 2016.

Yin, X., Dong, L., Wu, H., Ma, W., Zhang, L., Yin, W., Xiao, L., Jia, S., and Tittel, F. K.: Highly sensitive $\mathrm{SO}_{2}$ photoacoustic sensor for $\mathrm{SF}_{6}$ decomposition detection using a compact $\mathrm{mW}$-level diode-pumped solid-state laser emitting at $303 \mathrm{~nm}$, Opt. Express, 25, 32581-32590, 2017a.
Yin, X., Dong, L., Wu, H., Ma, W., Zhang, L., Yin, W., Xiao, L., Jia, S., and Tittel, F. K.: Ppb-level $\mathrm{H}_{2} \mathrm{~S}$ detection for $\mathrm{SF}_{6}$ decomposition based on a fiber-amplified telecommunication diode laser and a background-gas-induced high- $Q$ photoacoustic cell, Appl. Phys. Lett., 111, 031109, https://doi.org/10.1063/1.4987008, 2017b.

Zeninari, V., Kapitanov, V. A., Courtois, D., and Ponomarev, Y. N.: Design and characteristics of a differential helmholtz resonant photoacoustic cell for infrared gas detection, Infrared Physics and Technology, 40, 1-23, 1999.

Zheng, H., Lou, M., Dong, L., Wu, H., Ye, W., Yin, X., Kim, C. S., Kim, M., Bewley, W. W., Merritt, C. D., Canedy, C. L., Warren, M. V., Vurgaftman, I., Meyer, J. R., and Tittel, F. K.: Compact photoacoustic module for methane detection incorporating interband cascade light emitting device, Opt. Express, 25, 1676116770, 2017. 